\title{
Numerical Analysis of Ventilation for Ship E/R with CFD Method
}

\author{
Jianping Chen ${ }^{\mathrm{a}, *}$, Jie $\mathrm{Xu}^{\mathrm{b}}$, Litao Wang ${ }^{\mathrm{a}}$, Xinen Chen ${ }^{\mathrm{a}}$, and You Gong ${ }^{\mathrm{a}}$ \\ ${ }^{a}$ School of Ship Engineering, Guangzhou Maritime University, Guangzhou, 510725, China \\ ${ }^{b}$ Faculty of Automation, Guangdong University of Technology, Guangzhou, 510006, China
}

\begin{abstract}
The paper presents a CFD numerical simulation method for ship engine room ventilation. First, through the discretization of the fluid governing equations, apply the basic physical model of ship engine room established by GABIT Software to lay out the engine room outlet according to the air supply and then divide the meshes. After the physical model is established, import the FLUENT and then reasonably choose the boundary conditions, solving methods and solving precision. Finally, obtain the optimal scheme by the example of researching the airflow velocity, temperature and humidity distribution under different ventilation schemes, and compare the characteristics of various schemes. The method presented in the paper has a strong significance of theoretical analysis and practical guidance for optimizing the ventilation of the ship engine room.
\end{abstract}

Keywords: ship engine room; ship engine ventilation; numerical simulation; scheme optimization

(Submitted on December 12, 2017; Revised on January 13, 2018; Accepted on February 16, 2018)

(C) 2018 Totem Publisher, Inc. All rights reserved.

\section{Introduction}

The ship's engine room is the ship's main drive, which includes a host, boiler, booster units and other equipment. Compared with onshore machines and furnace room ventilation systems, ship engine ventilation has obvious characteristics. One of the greatest characteristics is that the ship engine room space is narrow, the unit volume of waste heat is 5 to 10 times the land $[7,8]$, the distribution of heating equipment is uneven, and the local regional temperature gradient is large. At the same time, with the development of science and technology, energy saving and environmental protection have become an important research direction of air conditioning technology [3].

In the application of ship engine room ventilation, there are comparatively less foreign literature, and domestic research is mainly centralized in universities and shipping research institute. Ventilation of the ship engine room is large space ventilation, which is similar to the ventilation system of thermal power stations and boiler room. But, the characteristic of the engine room ventilation itself determines the difficulty of ventilation design. Hao Weijian [2,5] organized numerical simulation for a container ship engine room airflow, which was calculated by the CFD software PHOENICS. In his paper, he analyzed the velocity field and the temperature field of the engine room and improved the ventilating condition by adjusting the air outlet, which concluded that the feasibility of ventilation design in the engine room by using numerical simulation. Zhou Shan [11] uses FLUENT to simulate the ventilation system of a passenger ship engine room. By analyzing the distribution of the internal temperature field and velocity field, he found that the temperature distribution in the engine room is not uniform, but changing the position and outlet angle can reduce the temperature of the pump area. Chen Ning, Zhang Dong [1] used CFD software AIRPAK to analyze a tanker ventilation system simulation. The detailed design and calculation of the ventilation system of the engine room given in his literature are of great reference value. As an appropriative HVAC airflow numerical simulation software, AIRPAK has simple design and requirement, and is more convenient than FLUENT as showed in the upper results. However, it has the limitation of building models and dividing meshes when the engine room is complicated. Suo Wenchao and Wang Xiancheng analyzed the three-dimensional temperature field and velocity field in the engine room and compared the experimental values. They think the numerical simulation result for engine room airflow is useful. By designing ventilation system for ship engine room, applying CFD

\footnotetext{
* Corresponding author.

E-mail address: jianpingchenjpc@ sina.com
} 
technology to do numerical simulation for engine room simulation and comparing each operating condition comprehensively, I obtained the optimal ventilation scheme, which provides the basis for the ventilation design. By researching the distribution of air velocity field, temperature field and humidity field and improving the air dead angles and vortexes, I make the engine room's temperature and humidity distribution more uniform. Meanwhile, I distributed the air reasonably, reduced the heat accumulation and exhausted the harmful gas in the engine room.

\section{Flow field basic control equation}

As a fluid in the pipeline and engine room, air follows the physical laws of certain air fluids, such as mass conservation, momentum conservation, energy conservation, etc. According to these laws and the law of actual physical state to establish a closed fluid motion basic equation, these equations, fluid flow state and law can be obtained. In this situation, the actual physical state can be laminar or turbulent, or component transfer or chemical reaction [4].

\subsection{Mass conservation equations}

The law of mass conservation is a universal law of nature which fluid flowing also follows. The law of conservation of mass can be expressed as: the mass added to the fluid micro component at any time is equal to the net mass that flows into the micro component at the same time. According to this law, we can get the mass conservation equation. According to this law, we can get the mass conservation:

$$
\frac{\partial \rho}{\partial t}+\frac{\partial\left(\rho u_{i}\right)}{\partial x_{i}}=0
$$

In Equation (1), $\rho$ is airflow density, $t$ is time, $U$ is flow velocity, $i$ 's are respectively the directions of coordinate axis $x, y$ and $z$. In the field of ventilation and air conditioning engineering, generally, air is incompressible fluid, that is, its density does not change with time. The continuity equation can be simplified as Equation (2):

$$
\frac{\partial u_{i}}{\partial x_{i}}=0
$$

\subsection{Momentum conservation}

In inertial coordinates, the equation can be expressed as:

$$
\frac{\partial\left(\rho u_{i}\right)}{\partial t}+\frac{\partial\left(\rho u_{i} u_{j}\right)}{\partial x_{j}}=-\frac{\partial p}{\partial x_{i}}+\frac{\partial \tau_{i j}}{\partial x_{j}}+\rho g_{i}+F_{i}
$$

In Equation (3), $p$ is static pressure, $\tau_{i j}$ is the shear stress, whose normal is $i$ direction and surface is $j$ direction. $\rho g_{i}$ is the volume force due to gravity in $i$ direction, $F$ includes other source terms. $\tau_{i j}$ is obtained by Equation (4):

$$
\tau_{i j}=\mu\left(\frac{\partial u_{i}}{\partial x_{j}}+\frac{\partial u_{i j}}{\partial x_{i}}\right)-\frac{2 \mu}{3} \frac{\partial u_{i}}{\partial x_{i}} \delta_{i j}
$$

In Equation (4), $\boldsymbol{\mu}$ is dynamic viscosity coefficient, The second term to the right of the equation takes into account the effect of volume expansion, and $\delta_{i j}$ is Kronecker function, when $i=j, \delta_{i j}=1$, when $i \neq j, \delta_{i j}=0$. 


\subsection{Energy conservation}

In the field of HVAC, the energy transfer form between the fluids is expressed by enthalpy $\left(h=\int_{298.15}^{T} c_{p} d T\right.$ ), where 298.15 is the reference temperature, the specific form is

$$
\frac{\partial(\rho h)}{\partial t}+\frac{\partial\left(\rho u_{i} h\right)}{\partial x_{i}}=\frac{\partial\left(k+k_{t}\right)}{\partial x_{i}} \frac{\partial T}{\partial x_{i}}+S_{h}
$$

In Equation (5), $k$ is the thermal conductivity of molecular motion, $k_{t}$ is the additional thermal conductivity of turbulence $\left(k_{t}=c_{p} \mu_{t} / \operatorname{Pr}_{t}, P \mathrm{r}_{t}\right.$ is turbulence Planck Number, $\left.P \mathrm{r}_{t}=0.85\right), t$ is temperature, $S_{h}$ is the source term, it can be any volume of heat source. The thermal conductivity of the solid region can be expressed as the following thermal conductivity equation.

$$
\frac{\partial(\rho h)}{\partial t}=\frac{\partial}{\partial x_{i}}\left(k \frac{\partial T}{\partial x_{i}}\right)+q_{v}
$$

In Equation (6), $q_{v}$ is the internal heat source.

Indoor air flow thermal and convective coexist, thereby Equation (6) and Equation (5) must be solved at the same time.

\subsection{Component transport equation}

The idea of solving component transport equation is to estimate the mass fraction $Y_{i}$ of each substance by the convectiondiffusion equation of the $i$ th substance. The component transport equation is expressed by the following general formula

$$
\frac{\partial\left(\rho Y_{i}\right)}{\partial t}+\nabla \cdot\left(\rho u Y_{i}\right)=-\nabla \cdot J_{i}+R_{i}+S_{i}
$$

In Equation (7), $R_{i}$ is the net production rate of this component due to chemical reactions, $S_{i}$ is the additional generation rate that results from the user-defined source term diffusion phase. When $N$ kinds of substances interact in the system, $(N-1)$ equations in this form must be solved. Because the sum of the mass fractions must be 1 , the fraction of the $N$ th substance equals 1 minus the sum of $(N-1)$ mass fractions solved.

(1) Mass Diffusion in Laminar Flow

In Equation (7), $J_{i}$ is the diffusion flux of substance, resulting from the concentration gradient. Diffusion flux of dilution approximation can be expressed as:

$$
J_{i}=-\rho D_{i, m} \nabla Y_{i}
$$

In Equation (8), $D_{i, m}$ is the diffusion coefficient of the $i$ th substance in the mixture. 
(2) Mass Diffusion in Turbulence

Mass diffuses in turbulence:

$$
J_{i}=-\left(\rho D_{i, m}+\frac{u_{t}}{S c_{t}}\right) \nabla Y
$$

In Equation (9), $S c_{t}$ is the turbulent Schmitt number (generally default to 0.7 ).

\section{(3) Substance Transporting in Energy Equation}

In the multi-component flow process, the diffusion of the component is accompanied by the energy transfer. For the enthalpy field, the diffusion is so significant that it cannot be simply ignored. Especially when the Lewis number of all substances is far greater than 1, ignoring it will result in serious errors.

$$
L e_{i}=\frac{k}{\rho c_{p} D_{i, m}}
$$

To sum up, the above conservation Equation (10) can be uniformly expressed as Equation (11).

$$
\frac{\partial(\rho \phi)}{\partial t}+\operatorname{div}(\rho u \phi)=\operatorname{div}(\Gamma \operatorname{grad} \phi)+S
$$

Its expanded form is

$$
\frac{\partial(\rho \phi)}{\partial t}+\frac{\partial(\rho u \phi)}{\partial x}+\frac{\partial(\rho v \phi)}{\partial y}+\frac{\partial(\rho w \phi)}{\partial z}=\frac{\partial}{\partial x}\left(\Gamma \frac{\partial \phi}{\partial x}\right)+\frac{\partial}{\partial y}\left(\Gamma \frac{\partial \phi}{\partial y}\right)+\frac{\partial}{\partial z}\left(\Gamma \frac{\partial \phi}{\partial z}\right)+S
$$

In Equation (12), $\phi$ is the solving variable which can represent $U, v, w$ and $T, \Gamma$ is the generalized diffusion coefficient, $S$ is the generalized source term.

For the equation to determine, $\phi, \Gamma$ and $S$ have particular forms. In ventilation and air conditioning engineering, air is generally handled as incompressible gas.Microscopically, distance of the same intermolecular remains constant.Macroscopically, the gas density $\rho$ does not change. In the equation group, $\frac{\partial \rho}{\partial t}=0$.

\section{Numerical solution method}

The control equation is a series of partial differential equations, whose solution method can be divided into analytic method, experimental method and numerical method. In general, it is very difficult to obtain the analytic solution of the system by analytic method. For some flow and heat transfer problems, experimental method and numerical method must be adopted. The solution of numerical method is to replace the field of the physical quantity (such as velocity field, temperature field, concentration field, etc.), which is continuous on the space coordinates, with a set of values of a finite number of nodes. The variables are linked by particular rules, which are a series of algebraic equations. The solutions of the algebraic equations are substituted for the approximate solutions of the variables. CFD model solution is to get the value of the variable by using numerical solution.

According to the difference of area, diffusion way of control equation and algebraic solution, there are mainly finite element method, finite analysis method and finite volume method to solve the numerical solution. Finite volume method is to divide the calculation domain into a series of continuous volume, and replace each control volume with a base point. The 
discrete equation derived by the finite volume method can guarantee the conservation properties. The discrete equation coefficients have definite physical meaning, which are widely used in the numerical calculation of fluid mechanics. The finite volume method is used for numerical analysis in the paper.

\subsection{Discretization of the calculation domain}

The discretization of the calculation domain is the basis of numerical calculation. Domain discretization is to divide the computing area into several sub-areas, and to determine the nodes of each area. This process is also called mesh generation. The discretization of calculation domain is to choose a finite number of discrete points to represent the original continuous space. The general implementation process is to divide the area into different non-overlapping but continuous sub-areas, and then determine the node position in each sub-area and the control volume represented by the node. After the regional discretization is completed, the following four geometric elements are obtained [9].

(1) Node: The geometrical position of the unknown physical quantity needed to be solved

(2) Control volume: the minimum geometric unit of the governing equation or conservation law

(3) Interface: it defines the interface's position of the control volume corresponding to each node

(4) Mesh lines: a curve cluster formed by connecting two adjacent nodes in the coordinate axis direction

In the simulation of ventilation problems, after the model is established, the model is divided into several control bodies. Select a node to replace each control body. The temperature, velocity and concentration of components on the control body are stored in the node.

\subsection{Discretization of governing equation}

The discretization of the governing equation is the transformation of the governing equations in the form of partial differentials into algebraic equations on each node. When the finite volume method is used, the physical quantity on the control body and its derivative need to be interpolated by the nodal physical quantity interpolation. Interpolation method is also known as discrete format. The discrete formats commonly used in finite volume are center differential method, first order upwind format, mixed format, exponential format, power format, second order upwind format and quick format. In the airflow area of engine room ventilation space, let arbitrary mesh nodes by E, W, S, N, T, B in the vertical intersection direction. In the airflow area of the engine room ventilation space, let an arbitrary mesh node be $\mathrm{p}$, and its adjacent mesh points in the intersecting perpendicular direction are called E, W, S, N, T, B. It is shown in Figure 1.

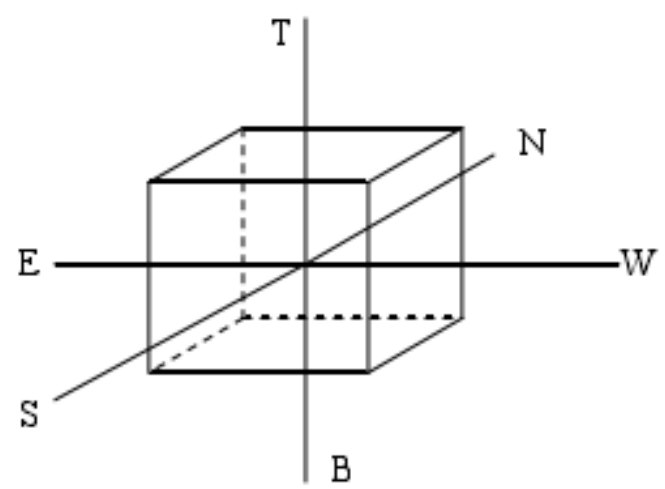

Figure 1. Control volume unit

In the three-dimensional steady-state coordinate system, the typical form of the finite-difference equation of the governing Equation (11) is expressed as Equation (13).

$$
a_{P} \phi_{P}=a_{E} \phi_{E}+a_{W} \phi_{W}+a_{S} \phi_{S}+a_{N} \phi_{N}+a_{T} \phi_{T}+a_{B} \phi_{B}+S_{c}
$$

Abbreviation: 


$$
a_{P} \phi_{P}=\sum a_{n b} \phi_{n b}+S_{c}
$$

In Equation (13) and Equation (14), $a$ is the coefficient of the difference equation, $S_{c}$ is the source term and establishes the independent variable required by the difference Equation (13) can be used to integrate the control volume $P$ in the calculation domain, and the general equation established by the control volume method can be obtained.

$$
\sum_{e, w, s, n, t, b}\left[\left(\rho u \phi-\Gamma_{\phi} \operatorname{grad} \phi\right) \times A\right]=\left(S_{\phi} V\right)_{P}
$$

In Equation (15), the sum of $e 、 w 、 s 、 n 、 t$ is the control volume's center of each interface, $V$ is the cubage of control volume, and $A$ is the absolute value for the control volume of an interface area, and its direction is the outer normal direction of the interface.

In Equation (15), both the convective terms and the diffusion terms are transformed into the values on the control volume interface, and the physical quantities at the interface are represented by the physical quantity of the nodes through interpolation. Different interpolation methods correspond to different discrete results. When establishing the discrete equation by the finite volume method, the continuity of the control volume interface must be maintained, the slope of the source term must be negative, and the center point coefficient must be equal to the coefficient of the adjacent point. Only by following the above principles can the results of the discrete equation reach the mathematical correctness and the physical rationality.

\section{Numerical solution process}

\subsection{Determination of boundary conditions}

FLUNET provides four types of boundary conditions: flow inlet and outlet boundary conditions, wall, repeatability and singular axis boundary conditions, and internal unit area and internal surface boundary [10]. The choice of boundary condition determines the convergence rate and greatly influences the calculation result. Therefore, the boundary condition should be ensured when the boundary conditions are set. A reasonable boundary type with good convergence should be selected. Model boundary conditions are set as follows:

\section{(1) Return air outlet by machine}

By the calculation of the previous air flow to determine a single outlet, it is easy to calculate the outlet air velocity. Therefore, for returning air outlet, the velocity inlet boundary conditions for incompressible flow is used, which the velocity, direction, temperature and mass fraction of the tuyere boundary are set, and the method of determining the turbulence condition adopts the hydraulic diameter $L$ and the turbulence intensity $I$. FLUENT default turbulence intensity can be a simple ventilation calculation, but to improve the accuracy of calculation, detailed calculation of the turbulence intensity value is needed. In the paper, the model's air outlet is regular. According to the definition of hydraulic diameter, which is four times of the ratio of the flow area to wet circumference, the hydraulic diameter of the air outlet is 0.2667 . In general, the outlet of indoor ventilation problems is a simplified model and the outlet velocity is relatively low, which belongs to moderate turbulence, whose intensity is generally between $1 \%-5 \%$. The specific value is calculated by the estimation formula, the formula is as follows:

$$
I=\frac{u^{\prime}}{\bar{u}}=0.16\left(\operatorname{Re}_{L}\right)^{-l / 8}
$$

In Equation (16), $u^{\prime}$ and $\bar{u}$ are the turbulence pulsation velocity and the average velocity, $l$ is the turbulence mixing length, the full development of the turbulence can take its hydraulic diameter, according to the hydraulic diameter and design of the outlet velocity calculated Reynolds number. For the full development of the turbulence, its desirable hydraulic diameter $L$ can be selected, $\operatorname{Re}_{L}$ is the Reynolds number obtained by the calculation of the hydraulic diameter $L$ and the outlet velocity.

(2) Wall boundary

In the case of thermal or convective heat transfer, the heat transfer conditions at the solid boundary are generally 
specified as follows: set the temperature distribution on the boundary stipulate the heat flow distribution on the boundary.

\subsection{FLUENT Solve parameter settings}

According to the above assumptions, the ".mesh" file of GAMBIT output is imported into FLUENT and FLUENT is set to solve the control parameters. The detailed steps are as follows:

(1) Check the mesh. Determine no negative mesh with no skewness, and is smooth the mesh with larger skewness. The minimum deviation offset of FLUENT is 0.8 , which can meet the requirement of calculation.

(2) Definition of the solver. In this paper, a pressure coupled solver developed by a separate solver is used. The default solution is adopted to solve the governing equations and the flow is a steady flow.

(3) The standard two-equation model is selected for the turbulence model. The wall function method is used for the near wall and the FLUENT default value is used for the coefficient of the system.

(4) Enable the component transport model. In this paper, a non-chemical reaction component transport model is used, in which the components in the air consisted of $\mathrm{H}_{2} \mathrm{O}$ (gas), $\mathrm{N}_{2}$ and $\mathrm{O}_{2}$.

(5) Operating environment settings. Set the standard pressure of 101325Pa, gravity acceleration of $-9.81 \mathrm{~m} / \mathrm{s}$, wet air, physical properties of water vapor parameters from the FLUENT directly call the database;

(6) Set the boundary conditions.

(7) Set the control method. The SIMPLE algorithm is used to solve the pressure - velocity coupling problem, set the relaxation factor of $\mathrm{H}_{2} \mathrm{O}$ at 0.7 and the other factors in the FLUENT default.

(8) Set monitoring residual to iteration after initialization calculation.

\subsection{Convergence determination and processing of results}

In the case of no requirement to calculate the number of steps, FLUENT default the calculation must meet the set of residual requirements to terminate; that is, residual is a convergence accuracy of the demarcation point. The actual residual is also set according to the specific situation. For the more complex flow field, the residual is set to be slightly larger, that is to say the accuracy of the convergence can be slightly smaller. But when the flow field is simple, the accuracy can be very high. In indoor ventilation engineering, the general residual should not be set less than 0.01 . In general, it is desired that the results of calculation can reach high precision, so the residual values are set as low as possible and the solution cannot reach the precision. At this time, the residual curve based on the representative variable of the flow field can be stabilized after several times of iteration, and it is considered that the residual curve has converged. The visual graphics derived from FLUENT can meet the general demands. However, in order to analyze the simulation results better, we need to use the post-processing software to obtain good results. In this paper, we use the TECPLOT to deal with the FLUENT graphics simple.

\section{Numerical simulation examples}

\subsection{Engine Room ventilation model}

Assume that the main equipment engine room includes the turbine, oil boiler, turbocharger, high temperature deaerator, plasma filter, part of the smoke pipe and so on. The compartment can be divided into three layers: the bottom layer of the pavement, along the height direction was the first and second grille layer, and the top deck layer where the outlets are arranged in the first, second grille layer below. There are many equipment and pipelines in the engine room, and the structure is complex. When the model is built, it must be simplified to make the model suitable for numerical simulation. The basic principle of the model simplification is:

(1) Regularize the devices with complex shapes and little effects on simplified simulation results. The turbine shape is very irregular, so drawing greatly increases the difficulty for dividing the meshes and calculation. Therefore, we simplify the shape into a regular one. Similarly, boiler, turbocharger units and ancillary equipment are replaced with regular body. 
(2) Remove the equipment pipeline that has little effects on air flow field. There are many pipelines in ship 30, including steam pipe, exhaust pipe and all kinds of water and electricity pipelines. It is impossible to list so many pipelines so this model lists only some of the steam turbine inlet steam pipe and some of the flue gas pipeline.

(3) Make the equipment surface a hydraulically smooth one. In ventilation numerical simulation, the viscous force of air and solid wall is often omitted. When the air flow goes through the wall, there is no friction loss. Therefore, when simplified, treat the engine room bulkheads and equipment, pipe wall thickness as hydraulically smooth surfaces.

(4) The treatment of air duct. In the engine room top, although the duct is in a larger size, it has no impact on the flow field in the lower engine room. The size of the duct is small, so the heat exchange inside and outside the duct is ignored and the duct in the model is simplified. In the outlet, the duct is substitute.

(5) The exhausting ventilation in the top deck of the upper deck has no effect on the flow field, so we replace it by the air surface.

\subsection{Influence of tuyere position on airflow}

By comparing the form of airflow organized, the ventilation mode that supplies air in the lower area is adapted. In order to make the air in the lower area flow evenly and rule out the heat of the lower part smoothly, we arrange outlets evenly in the lower part of the first and second grille. The position of the tuyere has a great influence on the airflow in the engine room. According to the principle of the tuyere arrangement, arrange more outlets near the boiler to ensure that there is abundant air to eliminate the heat. The area between the main engine and the boiler is an area workers come and go frequently. Therefore, increase the amount of air will make the staff more comfortable. When the host side of the space is relatively spatial and there is less cooling equipment, there are relatively fewer outlets. According to the air volume and the above arrangement, the initial location of the tuyere, the number and size of the specifications are as follows. The size of the tuyere is $0.4 \times 0.2 \mathrm{~m}$, and the air direction is vertical down. The tuyere arrangement is shown in Figure 2 .

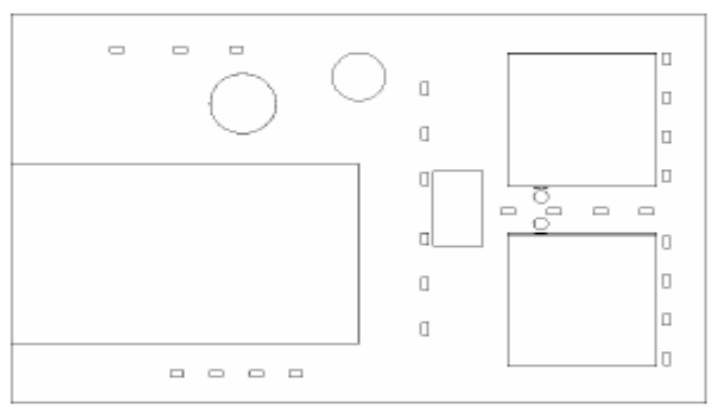

(a) The 1st floor

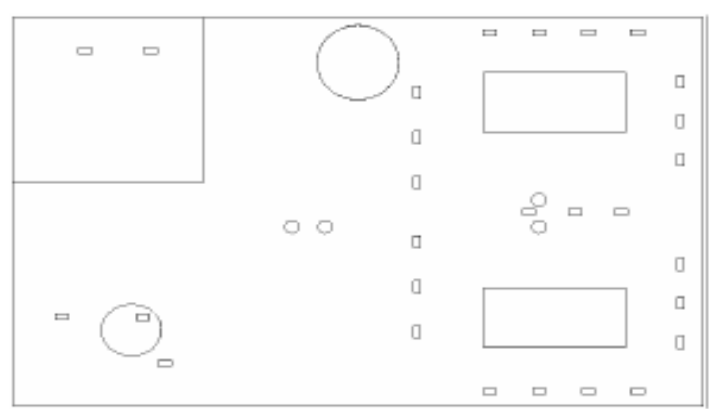

(b) The 2nd floor

Figure 2. The arrangement of the air inlet and outlet

According to the inlet and outlet arrangement, we select six ventilation schemes to simulate and analyze. In equation (1), a single air outlet in the middle of the top engine room is set to exhaust air mechanically. In Equation (2), a double air outlet is set and there is six-meter distance between two outlets. In Equation (3), four air outlets are all set in the top of the boiler and the status of side air supply outlet is controlled by the air valve. Specific scheme is shown in Table 1.

Table 1. The scheme of the air inlet and outlet

\begin{tabular}{|c|c|c|}
\hline Scheme & The status of side Air supply outlet & the form of air outlet \\
\hline 1 & Close & Single air outlet \\
\hline 2 & Close & Double air outlets \\
\hline 3 & Close & Four air outlets \\
\hline 4 & Open & Single air outlet \\
\hline 5 & Open & Double air outlets \\
\hline 6 & Open & Four air outlets \\
\hline
\end{tabular}

1. Velocity field analysis 
After obtaining the simulation results and the calculation of the heat load, $y=0 m$ section is in the right side of two boilers and the main engine. The area has the largest heat dissipation and is orthogonal to the return air outlet. The flow field is also the most complicated and the cross section is selected as the analytical plane. Regional air flow organization is established. Select sections of and $z=-3.5 m$ to analyze the lower part air flow in the control room. The selected crosssection position is shown in Figure 3.

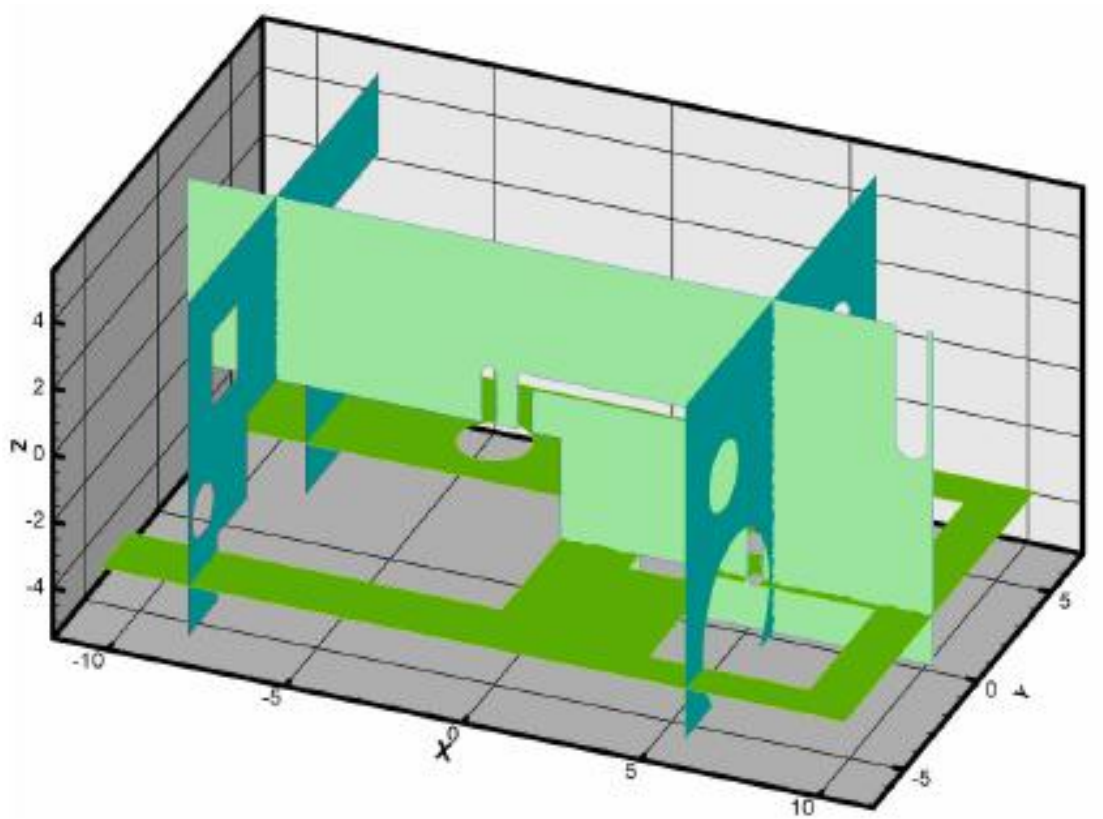

Figure 3. Selection of Analysis section

The velocity vector diagram shows the flow direction and velocity of the air, so the velocity vector diagram is used to analyze the airflow in the engine room. By analyzing the simulation results and changing the position of the outlets in the lower part of the control room makes little difference, the forms of airflow are similar with those of the velocity vector diagram. Therefore, we only chose $y=0 \mathrm{~m}$ section velocity vector diagram in scheme (1) and scheme (4) to analyze. Also, for $x=-8 m$ section and $z=-3.5 m$ section, we only chose scheme (3) and scheme (6) to analyze.

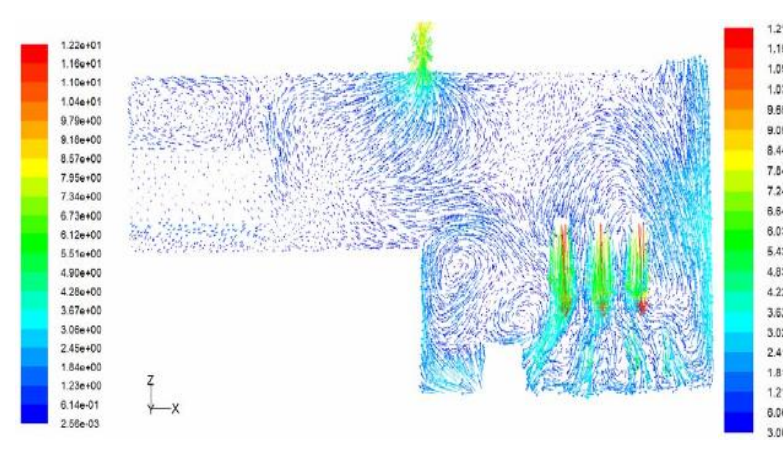

(a) Scheme 1

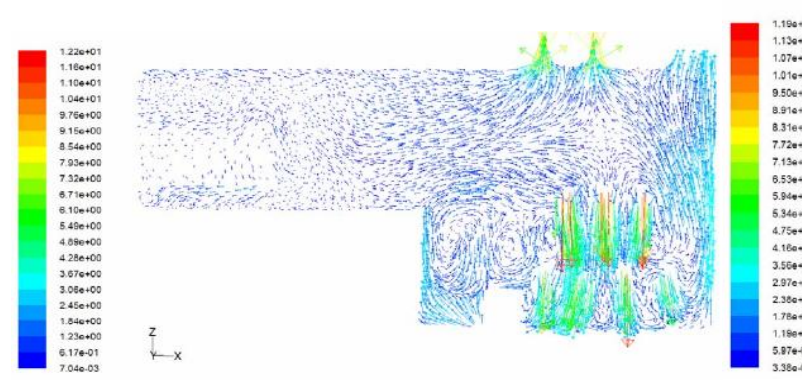

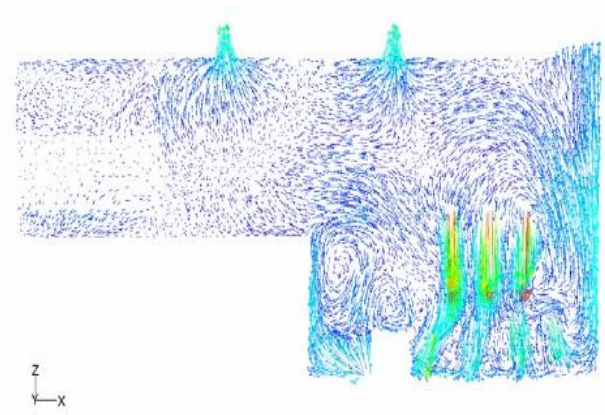

(b)Scheme 2

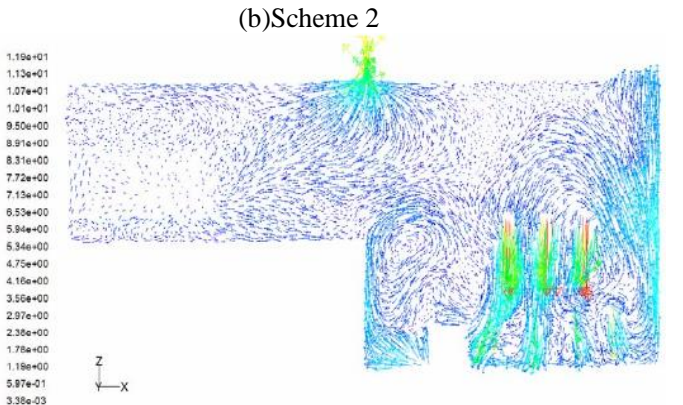


As showed in Figure 4, we can see that the air volume of each scheme is the same, and the airflow organizational form in the $y=0 \mathrm{~m}$ section is quite different. The concrete analysis is as follows:

(1) In scheme (1), because the single air outlet is in the middle of the top, except some air goes out from the right outlet, the air in the upper part flows to the middle of the top and the airflow velocity near the return air outlet is high; a large number of reflux on the right side of the boiler and the host appears.

(2) In scheme (2), double air outlet increases the air return area, but the overall return air volume remains the same. The air near the host can be effectively removed, while there is still a lot of reflux near the boiler side. Although the return air area greatly increased, its single return air volume reduced by half, resulting in less return air outlet than scheme (1), which makes the air in the top unable to go out and the air in the lower part unable to go to the top. The vents are much smaller, so that the gas rising to the top cannot be eliminated in time, while hindering the lower air rising to the top.

(3) In scheme (3), we consider the waste heat distribution in the entire engine room. Since the waste heat of the boiler side is about twice that of the host side, more outlets should be set on the boiler side. But no outlet is installed of the host side, causing air flow to the boiler side of the overall movement. Part of the air rises and discharges in the middle of the engine room, and part of the air flows to the boiler side and stops the rising air of the right host. Thus, a larger whirlpool in the middle of the engine room is formed.

(4) The airflow form of the scheme (4) is basically the same as the scheme (1). Similarly, scheme (5) and (6) is similar to the scheme (2) and (3), so we will not analyze in detail.

Through the above analysis, setting the air outlets at the top of the boiler side is conducive to the smooth elimination of the rising gas, while the overall structure of the air flow is also more reasonable. According to the above analysis, it can be seen that the return air outlet position should be determined by the distribution of air volume, so scheme (3) and scheme (6) have the best air return form.

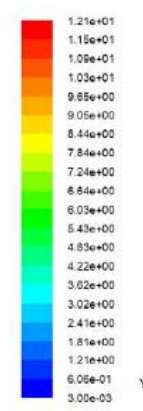

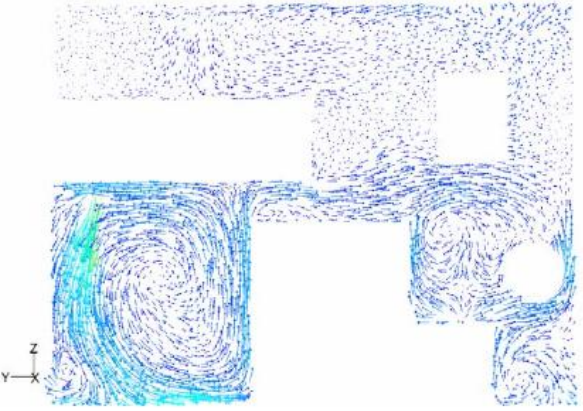

(a) Scheme 3

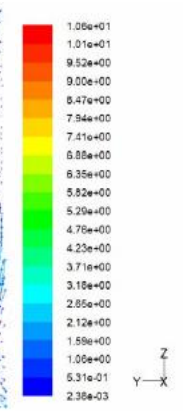

Figure 5. The velocity field cloud image while $x=-8 m$

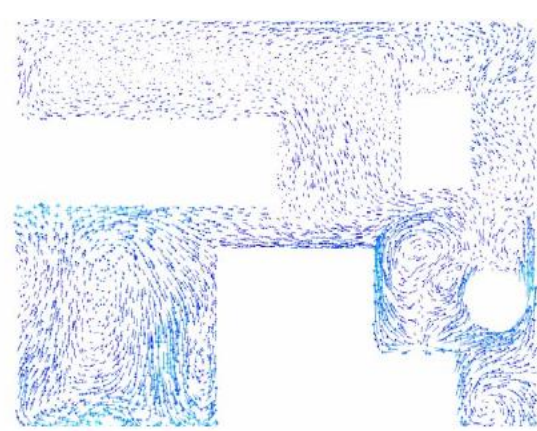

(b)Scheme 6
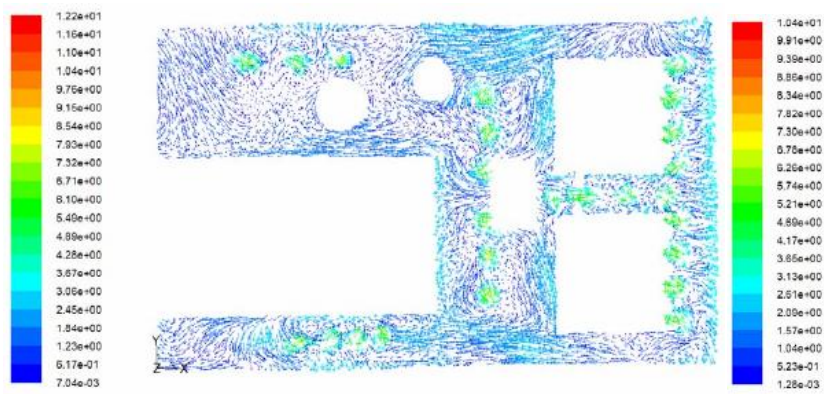

(a) Scheme 3

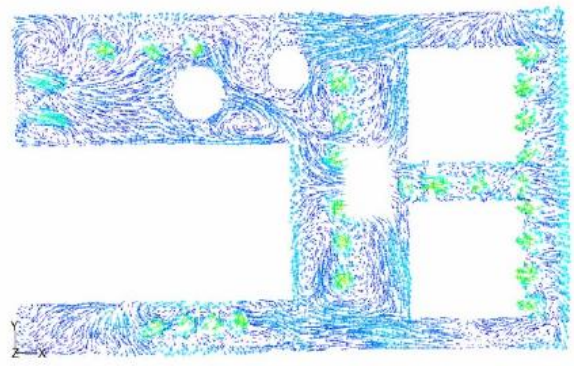

(b) Scheme 6

Figure 6 . The velocity field cloud image while $z=-3.5 \mathrm{~m}$

Chose $x=-8 m$ section and $z=-3.5 m$ section to analyze the influence of side outlet on airflow. From Figure 5(a), in scheme (3), there is a huge vortex in the lower part of the control room. The vortex starts from the left side of the bulkhead, rises along the wall of the host and flows back to the outlet. As can be seen from the vector diagram, the velocity 
of the air is gradually reduced from the whirlpool boundary to its center. In Figure 6(a), the air in the lower part of the control room flows more to the host side, and moves less in the middle. That is, the whirlpool is in a relatively static state. Most of the airflow rotates in the lower part of the control room, which causes air replacement. Through analysis, the more reasonable form of airflow in this area is that the air in lower part of the control room bypasses the bottom and rises to the top. From Figure 5(b) and Figure 6(b), it can be seen that after increasing the oblique air outlet, the airflow in the control room has obviously changed. The large whirlpool disappears because the disturbance of the side air supply increases the airflow to the middle of the engine room. The small whirlpool in the lower part of the control has little impact on the overall air flow. Therefore, scheme (6) has better effects than scheme (3) on the air replacement in the control room.

\section{Temperature field analysis}

The form of airflow is the basis of the existence of other fields (temperature field, humidity field, etc.) in the ventilation space. From the form of airflow, scheme (6) is most reasonable. In order to get a reasonable air outlet scheme, further verification in the temperature field is needed. We chose $y=-0.5 m$ and $x=6 m$ cross-section to analyze the temperature distribution.

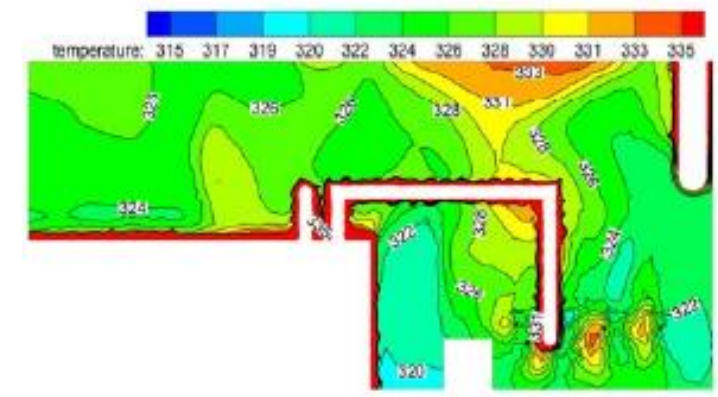

(a) Scheme 1

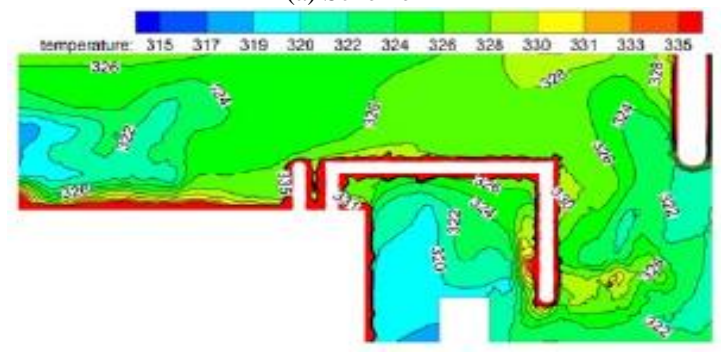

(c) Scheme 3

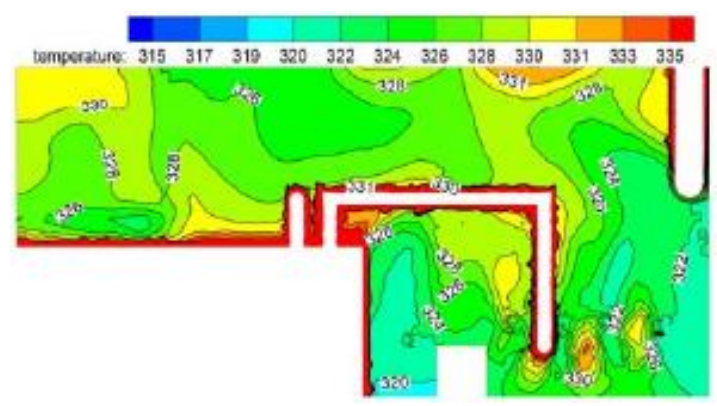

(e) Scheme 5

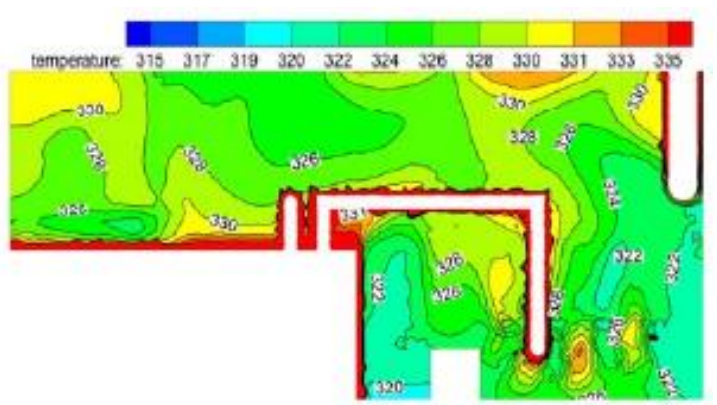

(b)Scheme 2

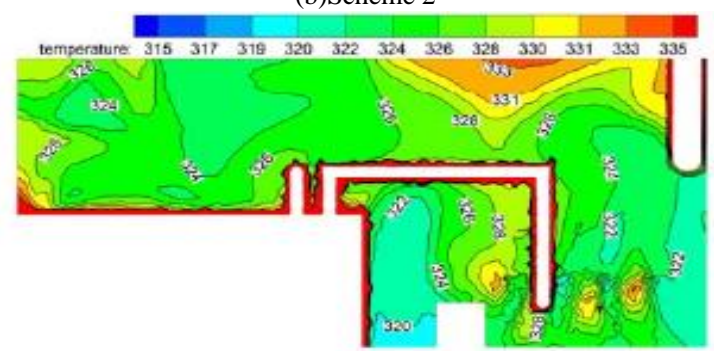

(d)Scheme 4

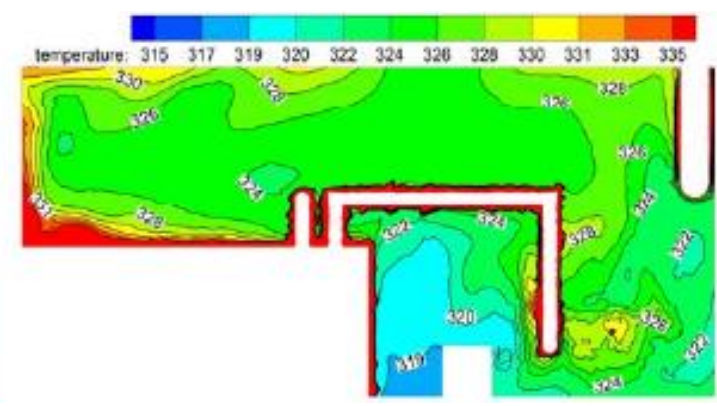

(f)Scheme 6

Figure 7. The temperature distributing cloud image while $y=-0.5 \mathrm{~m}$

From Figure 7, except the top of the boiler, the influence of the temperature distribution in the six schemes is not obvious at all. There is little influence on the temperature after increasing the lateral tuyere at the lower part of the control room. Therefore, we can analyze the influence of the position of the return air outlet and the side air outlet.

(1) Chose scheme (4) and scheme (6) to analyze the influence of the position of the return air outlet. Compared to scheme (6), there is a wide high temperature area in the top of the boiler in scheme, while the temperature difference of the other parts of is small, indicating that the temperature of the engine room is less affected by the return air position. Though there is no return air outlet in scheme (6), the high temperature airflow in the top of the boiler is effectively controlled. The 
airflow rising from the boiler to the top of the engine room is at high temperature. As the heat dissipation from the boiler surface is large and the airflow rising along the boiler wall takes a lot of waste heat. When the high temperature gas reaches the top of the engine room to form a high temperature area, this part of the high temperature gas not only affects the overall temperature distribution, but also may do harm to the equipment and personnel safety. So, it must be promptly excluded. According to the analysis of the airflow organization, the high temperature region of scheme (4) is far from the return air outlet, and the return mode of scheme (6) focuses on controlling the airflow at the top of the boiler. So, the effect is much better than that in scheme (3) because the range of high temperature is significantly reduced. It is shown in Figure 8.

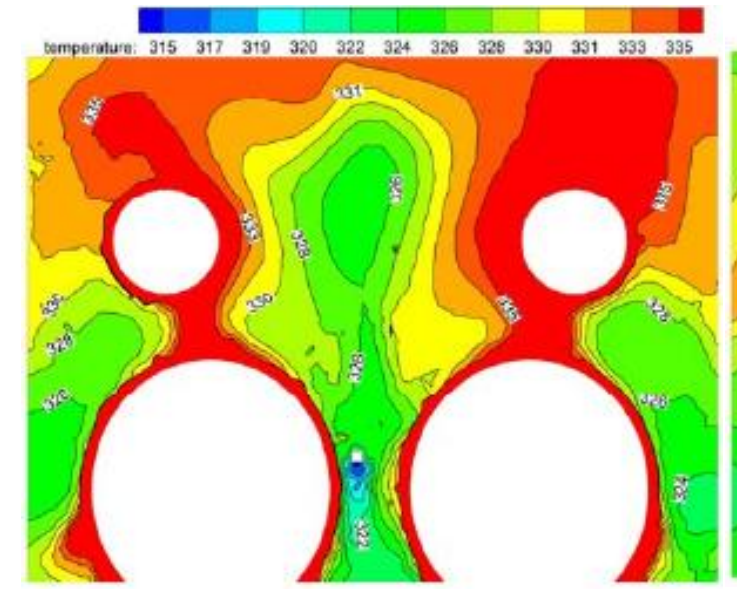

(a) Scheme 4

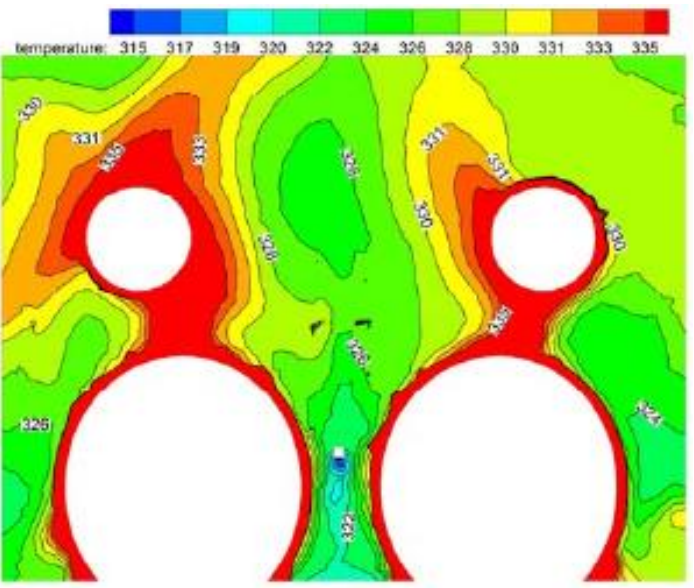

(b) Scheme 6

Figure 8. The temperature distributing cloud image while $x=6 \mathrm{~m}$

(2) Scheme (3) and (6) were used to study the influence of adding outlets at the lower side of the control room on the area.

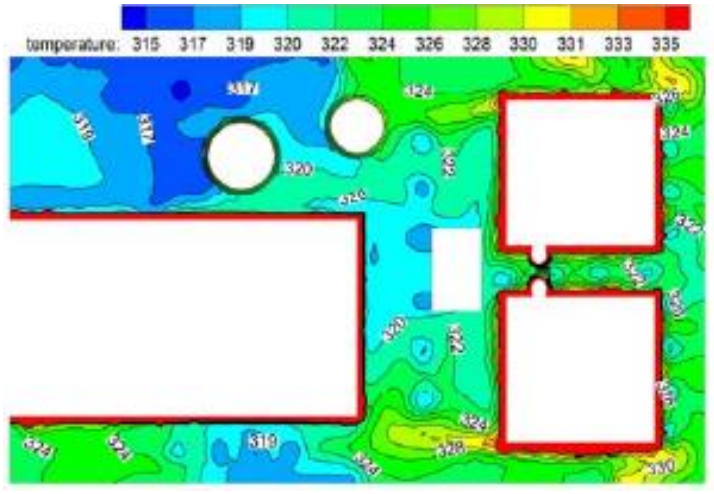

(a) Scheme 3

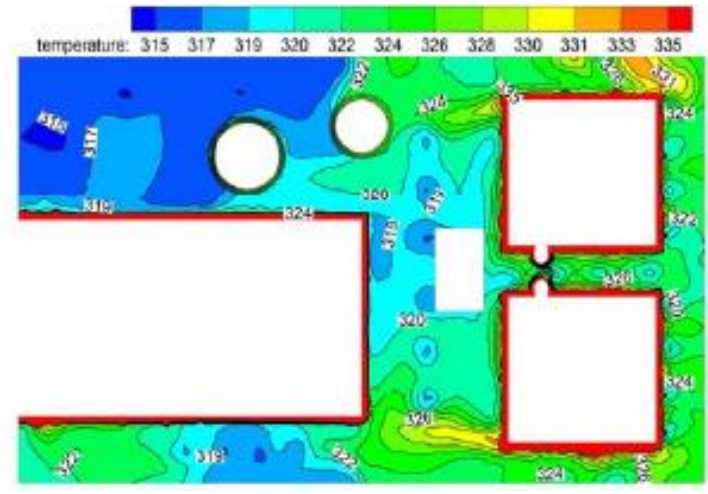

(b)Scheme 6

As showed in Figure 9, after the lateral tuyere is opened, the average temperature in the lower part of the control room is reduced and the temperature distribution is more uniform. In the scheme (3), a small area to the left of the engine room is formed. It is found that the airflow condition is poor in the area, which causes some air are unable to be normally discharged and the temperature is slightly higher than the surrounding environment. In summary, a rational arrangement of the air flow organization can effectively exclude waste heat. Through analysis, the position of the return air outlet has little effect on the airflow in the middle and lower part of the engine room. Adding the oblique air outlet only has a good effect in the local area. Through comparative analysis, the airflow organization of scheme (6) is more reasonable.

\subsection{Improvement of partial airflow}

The airflow distribution in the engine room is complicated, so there will always be dead ends and whirlpool in the ventilation. However, dead ends and whirlpool of security risks or large impact on flow fields must be avoided. In the assumption that the ventilation mode is fixed, there are two methods to eliminate the air dead ends and whirlpool. First, set obstacles. The effect of obstacles on indoor airflow is very large, and this method is rarely used in practical engineering because it increases unnecessary obstacles to the ventilation space [6]. Second, induced ventilation. Set inducing outlet of a 
dead zone or swirl areas, and re-adjust the ventilation system. The method has a significant effect in small ships. Due to the high wind speed, it is generally combined with the use of ventilation systems, and it is seldom used in modern ships.

In the paper, air flows from the bottom to the up, and the wind surface of obstacles has no staying area. Considering the economic costs, install a screen [3] at the right side of the host as an obstacle to eliminate this whirlpool. The screen's position is shown in Figure 10.

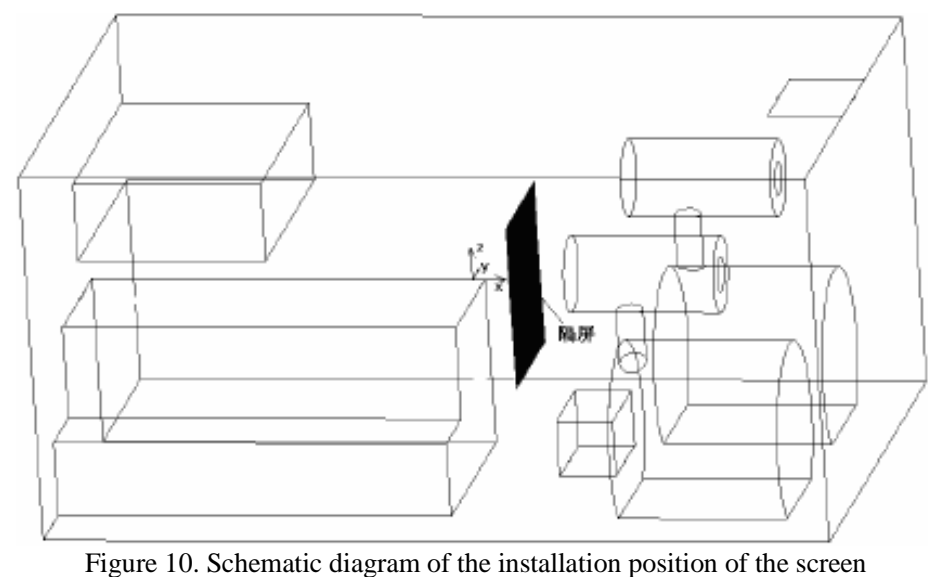

The screen is $4 \mathrm{~m}$ wide, same as the host right width, and $5 \mathrm{~m}$ high. The bottom is above the grid, and the height is beyond the second floor. This height is the also the same as the swing zone of the whirlpool. Airflow will collide with the obstacle. Select the condition of the air temperature $37{ }^{\circ} \mathrm{C}$, and air supply speed of $9.4 \mathrm{~m} / \mathrm{s}$ to simulate the impact of the screen. Then chose $y=-0.5 \mathrm{~m}$ section of the situation to analyze. The distribution of airflow after installation of the screen is shown in Figure 11.

Figure 12(b) is the velocity vector of the host's right side after the installation of screen. Compared with Figure 12(a), because of the screen, the airflow of the upper part rises from left to right and the airflow of the right-side exhausts directly from the outlet when reaching the top of the engine room. Therefore, the whirlpool of the screen position is eliminated, and the return air on the right side of the screen is significantly reduced.
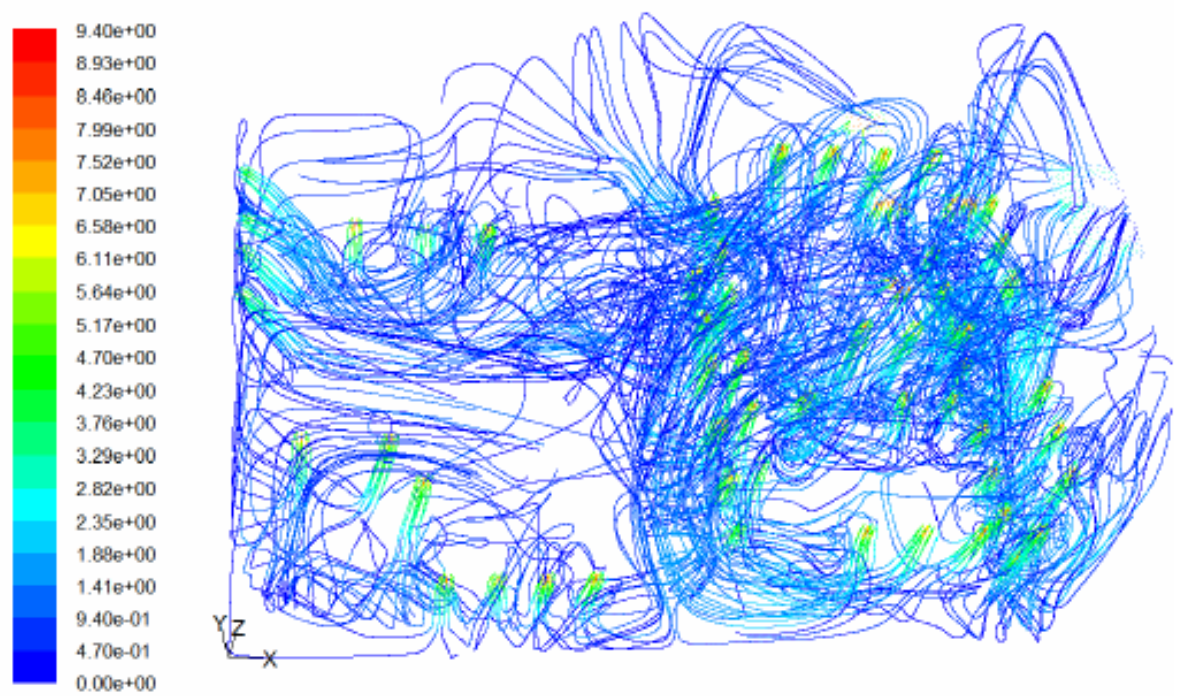

Figure 11. Air flow chart in engine room

Due to the screen's obstruction, the inter-boiler airflow is changed. As showed in Figure 13 (b), the inter-boiler airflow is brought together in the middle of the engine room and rises to the top before the boiler console. The airflow from the right bulkhead produces a small amount of reflow but does not produce a large whirlpool. After a short distance, it converges with other rising air and the problem of heat accumulation will not occur. Figure 13 shows the role of the screen in the 
ventilation system. Its impact on the air is very large. As long as the conditions permit, a reasonable set of screening can effectively improve the airflow in some areas of the organization.

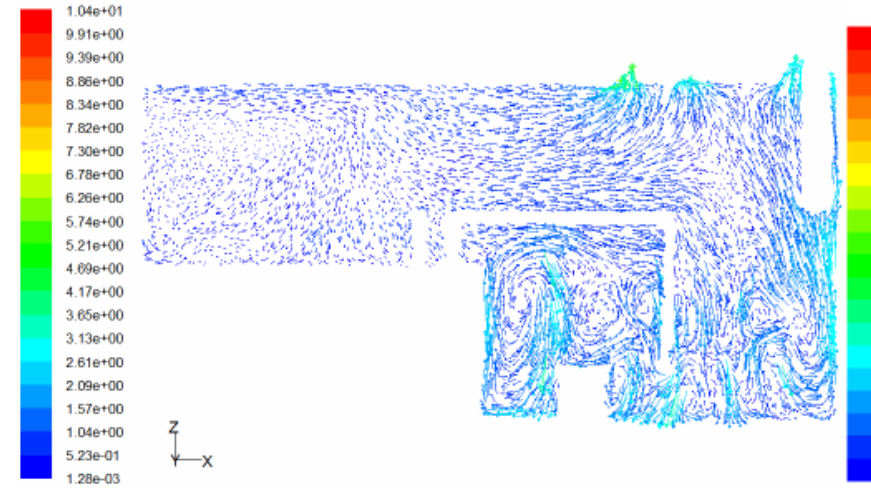

(a) Before improvement

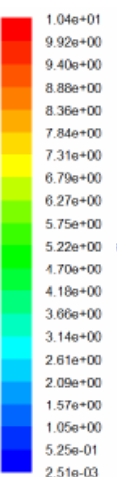

$\begin{array}{ll}\text { (a) Before improvement } & \text { (b)After improvement } \\ \text { Figure 12. Comparison of the airflow distribution before and after the improvement }\end{array}$

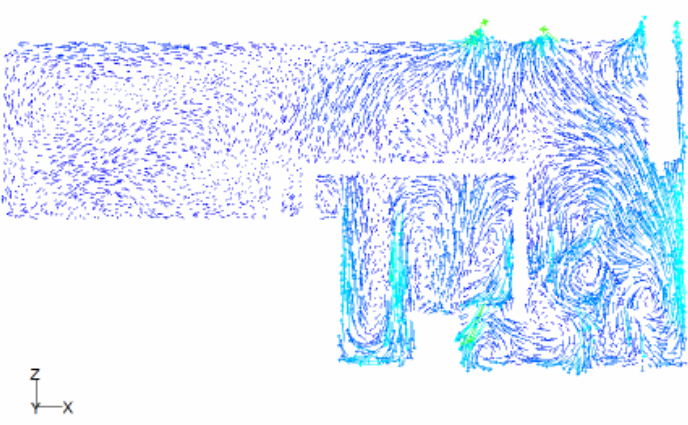

Figure 13 the screen temperature distribution on the right side of the host before and after the installation of the screen. From the comparison of Figure 13(a) and (b), it can be seen that the temperature on the right side of the screen is much higher than that before installation. The temperature difference on both sides of the screen increases, indicating that the screen has played a role in heat insulation, and effectively prevents the high temperature air flow by the boiler side to flow to the host side and the personnel region. The effect is obvious.

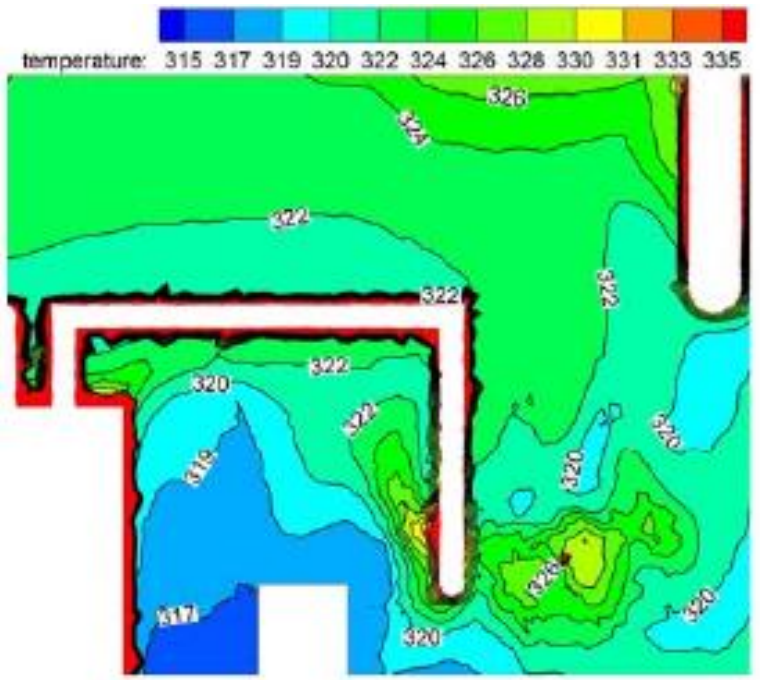

(a) Before improvement

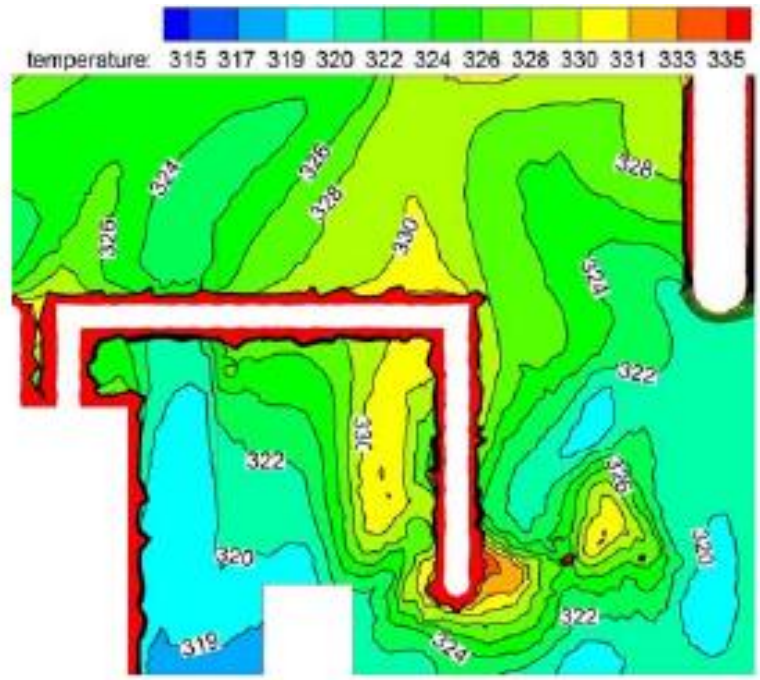

(b)After improvement

Figure 13. The temperature distribution of the right side of M/E before and after the improvement

According to the above analysis of velocity field and temperature field, the form of airflow changed after the screen was installed. The whirlpool is eliminated, the overall airflow distribution is more reasonable, and the temperature distributing caused by the airflow can benefit the elimination of the boiler's waste heat and can prevent the heat from spreading to the host. The installation location and size of the screen should be selected properly otherwise it will affect the air distribution of the entire engine room. Adding screen can improve the airflow form of the local area. In order to better observe the inside environment, we chose the working condition of $x=7 \mathrm{~m}$ and $z=-1 \mathrm{~m}$ sections with added screens for study. Figure 14 and Figure 15 respectively reflect the distribution of the velocity field and the temperature field in the boiler area and the central area of the engine room.

In regard to the above analysis, the change of the relative humidity and the temperature distribution is basically the same. Shown in Figure 16, the temperature of the lower part of the control room is lower and its relative humidity is higher. In general, the lower the temperature, the higher the relative humidity. 

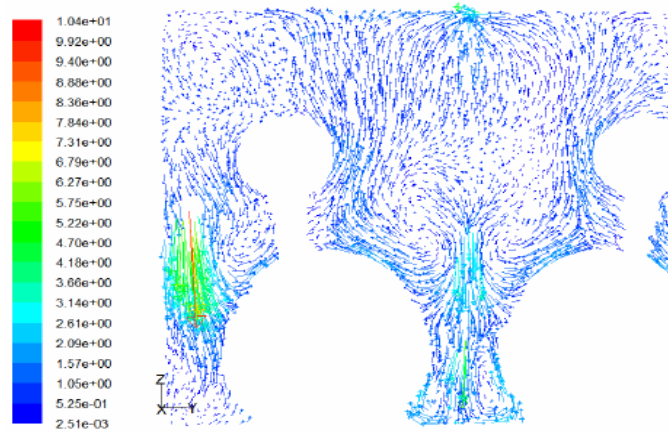

(a) Velocity field distribution

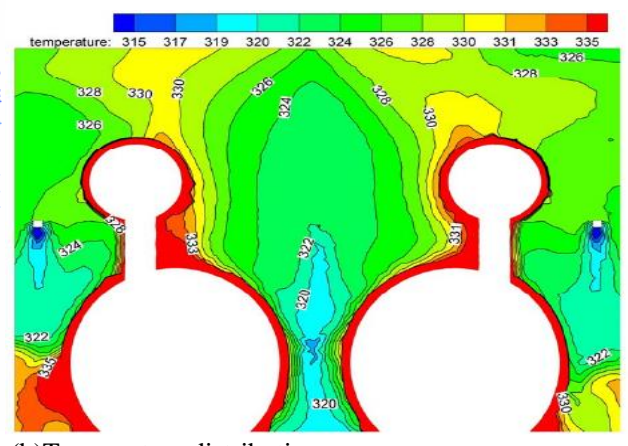

(b)Temperature distribution

Figure 14. Velocity field and temperature field distribution in section $x=7 \mathrm{~m}$
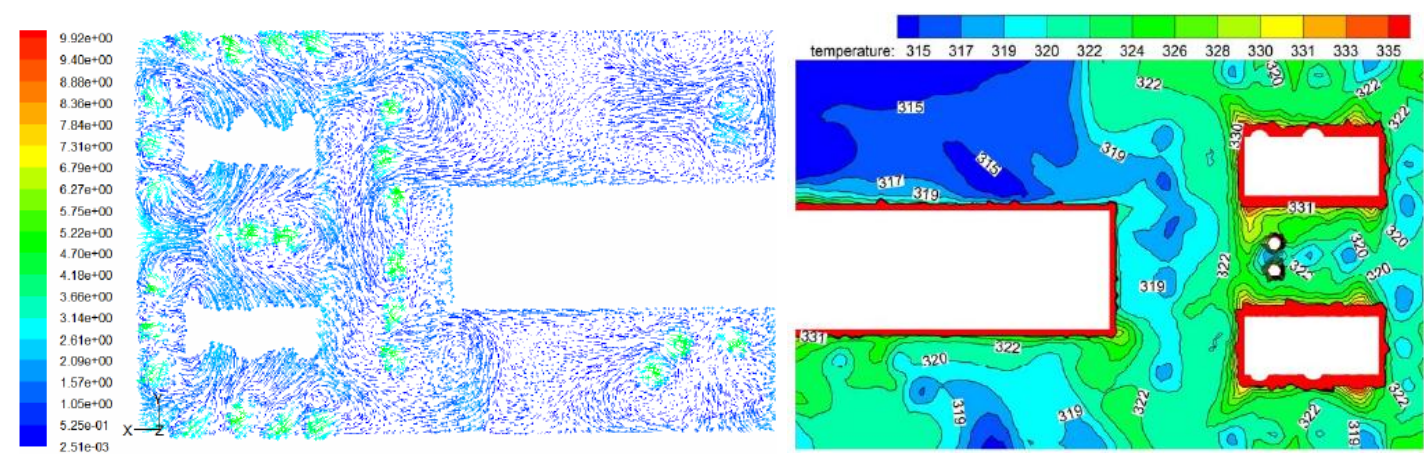

Figure 15. Velocity field and temperature field distribution in section $z=-1 \mathrm{~m}$

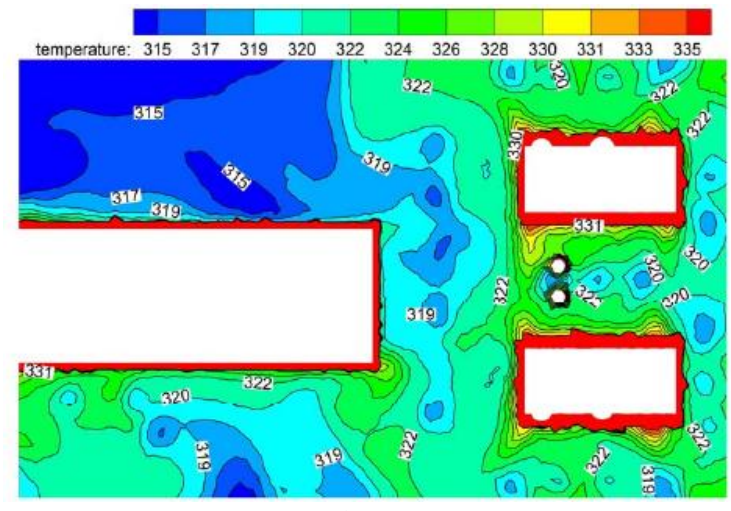

(a)In section $x=7 \mathrm{~m}$

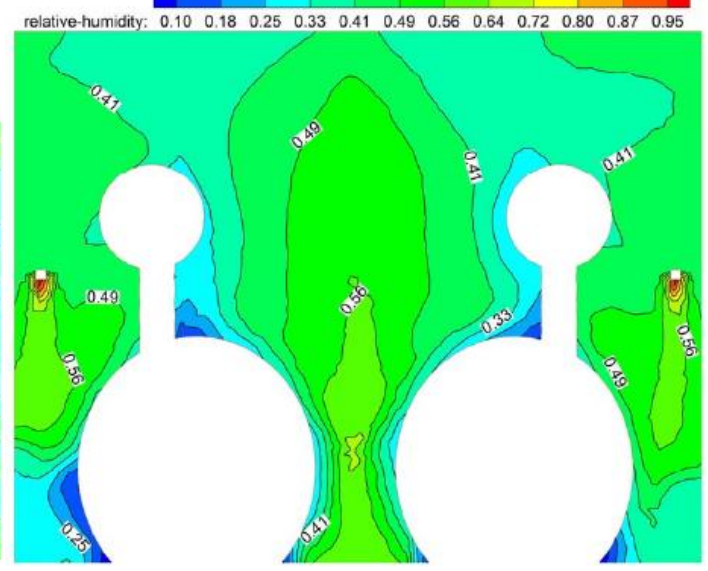

(b)In section $z=-1 m$

Figure 16. Relative humidity field distribution digraph

\section{Conclusions}

In this paper, the basic controls equation and discretion of air fluid in the engine room is presented. CFD method is used for numerical analysis and simulation. By comparing six schemes of different outlets arrangement, it is most reasonable to arrange the outlets at the top of the boiler. Moreover, partial airflow is significantly improved after adding side tuyere at the lower part of the control room. The air supply parameter has a larger influence on the E/G's environment. When the air supply velocity is fixed, the air supply temperature determines the overall temperature, but has little influence on the inside air speed and the relative humidity. With fixed air supply temperature, different velocity has relatively large influence on the whole engine room. At last, the ventilation condition is specifically analyzed. We know that in case of meeting the requirements of the engine room, reasonable ventilation can save ventilating energy. Setting screen can solve the problem of host whirlpool and insulate heat effectively. 


\section{Acknowledgements}

This work is partially supported by Guangdong Natural Science Foundation (Project No: 2014A030313792 and No.: 2016A030313125) and Guangdong Traffic Science and Technology (Project No: 2015-02-050). It is also supported by the China Postdoctoral Science Foundation (Project No: 2015M581622).

\section{References}

1. N. Chen, D. Zhang, "Calculation of Mechanical Ventilation in Ship Engine Room and Analysis of Airflow”, Ship Science and Technology, vol.31, no.3, pp. 73-76,2009

2. W. J. Hao, Y. X. Wang, "Numerical Simulation of Temperature Field and Velocity Field in The Engine Room", Journal of Dalian Maritime University, vol.31, no.1, pp.39-41.68,2005

3. X. Huang, "Air Conditioner Engineer", Chinese Machine Press, pp.6-7,2006

4. F. Jiang, "FLUENTAdvanced Application and Case Analysis", Beijing: Tsinghua University Press, pp. 7-45, 2008.

5. F. G. Liu, X. S. Meng, Y. Zhang, "Numerical Simulation of Thermal Environment in Ship Engine Room", Journal of Dalian Maritime University, vol.37, no.2, pp.136-138,2011

6. D. H. Qi, "Influence of Indoor Obstruction on Airflow Structure in Embedded Air - Conditioning Room”, Shanghai Jiao Tong University, pp.60-62,2009

7. H. Sha, "Ventilation of Engine Room, Chinese Version Translated by Lin Ruidong", Renmin Press, pp.89-124,1956

8. W. C. Suo, X. C. Wang, "Numerical Simulation of Air Flow Field in Boat Engine Room", Ship Science and Technology, vol.30, no.1, pp. $149-152,2008$

9. W. Q. Tao, "Numerical Heat Transfer (2nd Edition)”, Xi'an: Xi'an Jiaotong University Press, pp. 483-487, 2001

10. Y. Yu," FLUENT Getting Started with Advanced Tutorials", Beijing: Beijing Institute of Technology Press, pp. 236-258,2008

11. S. Zhou, "Numerical Simulation of Thermal Environment of Diesel Engine", Dalian Maritime University, 2010.

Jianping Chen received his B.S. degree in College of Shipbuilding Engineering from Harbin Engineering University, M.S. Degree in School of Naval Architecture, Ocean \& Civil Engineering from Shanghai Jiaotong University, and PH.D. Degree in School of Civil Engineering and Transportation from South China University of Technology. His research interests include ship performance research and ship structure analysis. 